\title{
MicroRNA-155 promotes the ox-LDL-induced activation of NLRP3 inflammasomes via the ERK1/2 pathway in THP-1 macrophages and aggravates atherosclerosis in $\mathrm{ApoE}^{-/-}$mice
}

\author{
Ruihua Yin ${ }^{1}$, Xiaoyan Zhu ${ }^{2}$, Jing Wang ${ }^{1}$, Shaonan Yang ${ }^{1}$, Aijun Ma ${ }^{1}$, Qi Xiao ${ }^{1}$, Jinyang Song ${ }^{1}$, \\ Xudong $\operatorname{Pan}^{1}$ \\ ${ }^{1}$ Department of Neurology, ${ }^{2}$ Department of Critical Care Medicine, The Affiliated Hospital of Qingdao University, Qingdao University, Qingdao \\ 266100, China \\ Contributions: (I) Conception and design: R Yin, A Ma, S Yang; (II) Administrative support: X Pan, A Ma; (III) Provision of study materials or patients: \\ R Yin, X Zhu, J Wang; (IV) Collection and assembly of data: R Yin, Q Xiao, J Song; (V) Data analysis and interpretation: R Yin, X Zhu, J Wang; (VI) \\ Manuscript writing: All authors; (VII) Final approval of manuscript: All authors. \\ Correspondence to: Dr. Xudong Pan; Dr. Aijun Ma. Department of Neurology, the Affiliated Hospital of Qingdao University, No. 59 Haier Road, \\ Qingdao, Shandong 266100, China. Email: drpan022@163.com; drmaj@126.com.
}

\begin{abstract}
Background: Nucleotide-binding oligomerization domain-like receptor protein 3 (NLRP3) inflammasome activation can induce the secretion of IL-1 $\beta$ and IL-18 and after promoting the development of atherosclerosis. MiR-155 is an important microRNA that modulates inflammation in atherosclerosis, but the role of miR-155 in the regulation of the NLRP3 inflammasome is still unknown.

Methods: The atherosclerosis model was set up using $\mathrm{ApoE}^{-/-}$mice, and the lentiviral vector (LV) was used to interfere the expression of miR-155. HE stains was used for plaque morphology, immunohistochemistry (IHC) and western blot were used for protein expression quantification. We used oxidized low-density lipoprotein (oxLDL) to incubate PMA-preprocessed THP-1 macrophages and detected NLRP3 inflammasome activation and ERK1/2 phosphorylation by western blot and Enzyme-linked immunosorbent assay.

Results: HE stains showed that the intravascular plaques in the miR-155-up group were remarkably increased, compared with negative control (NC) group. Results of IHC showed that the expression of caspase-1 and IL-1 $\beta$ in the miR-155-up group was the highest of four groups, consist with the Western blot analysis. The results of in vitro experiment show that ox-LDL promoted NLRP3 inflammasome activation and ERK1/2 phosphorylation. Blocking the ERK1/2 pathway could inhibit ox-LDL-induced NLRP3 inflammasome activation. Moreover, we found that the overexpression of miR-155 promoted the activation of the ox-LDL-induced NLRP3 inflammasome, which could also be blocked by the ERK inhibitor U0126.

Conclusions: MiR-155 aggravates the carotid AS lesion in $\mathrm{ApoE}^{-/-}$mice and exerts a regulatory effect on NLRP3 inflammasome activation in ox-LDL-induced macrophages via the ERK1/2 pathway.
\end{abstract}

Keywords: Atherosclerosis; oxidized low-density lipoprotein (ox-LDL); NLRP3 inflammasome; miRNA-155; ERK1/2

Submitted Sep 06, 2019. Accepted for publication Sep 27, 2019.

doi: 10.21037/apm.2019.10.11

View this article at: http://dx.doi.org/10.21037/apm.2019.10.11

\section{Introduction}

Inflammation occurs throughout the occurrence and development of atherosclerosis (AS) (1-3), which eventually leads to plaque rupture and thrombosis (4).
Monocytes, important immune cells in AS lesions, accumulate under vascular endothelial cells and turn into macrophages through the phagocytosis of oxidized low-density lipoprotein (ox-LDL). Macrophages release 
inflammatory cytokines such as interleukin- $1 \beta$ (IL-1 $\beta)$ and subsequently activate a cascade of other proinflammatory cytokines that aggravate vascular inflammation and promote AS progression (5). The activation of the NLRP3 inflammasome is an important source of IL-1 $\beta$ involved in the AS inflammatory response, which can be stimulated by various signals, such as ox-LDL and lipopolysaccharide (LPS) $(6,7)$. The NLRP3 inflammasome is composed of nucleotide-binding oligomerization domain-like receptor protein 3 (NLRP3), caspase-1, and apoptosis-associated speck-like protein containing a caspase recruitment domain protein (ASC). These NLRP3-related proteins were reported to be highly expressed in the plaque tissues of AS patients, especially in unstable plaques. The expression levels and the degree of vascular stenosis showed a positive correlation with AS clinical risk factors (smoking, hypertension, diabetes, hypercholesterolemia, etc.) (7-9). These findings suggest that the NLRP3 inflammasome is involved in the generation of AS plaques and is strongly associated with plaque instability.

As a multifunctional microRNA, miR-155 is an important molecule that regulates the inflammatory signaling pathway of AS $(10,11)$. It has been found that the expression of miR-155 in the plaques or plasma of AS patients and mice are significantly increased and are accompanied by the dysregulation of various inflammatory factors (11-15). It was reported that the expression of NLRP3 inflammasomerelated proteins, such as $\mathrm{P} 2 \mathrm{X}$ purinoceptor 7 ( $\mathrm{P} 2 \mathrm{X} 7)$, caspase- 1 and IL- $1 \beta$, was down-regulated in miR- $155^{-/-}$ dendritic cells (DC); this was accompanied by a decrease in the phosphorylation of ERK1/2 (16). These findings show that miR-155 may be associated with the activation of the NLRP3 inflammasome, but whether ERK1/2 was the downstream pathway activated by miR-155 was not confirmed. Other studies found that miR-155 can activate NF- $\mathrm{KB}$ through the ERK1/2 pathway in vascular smooth muscle cells (VSMCs) (14); NF- $\kappa B$ is the master switch that promotes the expression of NLRP3-related proteins and of the IL-1 $\beta$ and interleukin-18 (IL-18) precursor proteins during the priming of the NLRP3 inflammasome (17). Therefore, we hypothesized that miR-155 could affect the activation of the NLRP3 inflammasome via this ERK1/2NF- $\kappa \mathrm{B}$ pathway. In this study, we verified the effect of miR155 on the carotid AS lesions in $\mathrm{ApoE}^{-/-}$mice. Then we used ox-LDL-induced THP-1 cells as the AS inflammation model and detected the activation of ERK1/2, NF- $\mathrm{BB}$, and NLRP3 inflammasome to clarify the miR-155 regulation of the NLRP3 inflammasome and to determine its mechanism.

\section{Methods}

\section{Antibodies and reagents}

The anti-NLRP3, anti-caspase-1, anti-IL-1 $\beta$, and antiIL-18 antibodies used in the present study were bought from Abcam (Cambridge, UK). The anti-ERK1/2, antiphospho-ERK1/2 (Thr202/Tyr204), anti-NF-кB (p65) and anti-phospho-NF- $\kappa \mathrm{B}$ (p65) antibodies in the present study were bought from Cell Signaling Technology (Boston, USA). The microRNA mimics, inhibitors, and negative controls (NC) were obtained from GenePharma (Shanghai, China). The LV-miR-155 and LV-miR-155-RNAi were bought from HanHeng Biology (Shanghai, China).

\section{Animals}

Forty male 6-week-old male ApoE $\mathrm{E}^{-/-}$mice (in C57BL/6J background) were bought from Vital River (Beijing, China). Forty mice were randomly divided into 4 groups (10 mice in each group): blank group (AS mice without any transfection), NC group (AS mice transfected with LVNC), miR-155-up group (AS mice transfected with LVmiR-155), and miR-155-down group (AS mice transfected with LV-miR-155-RNAi). AS group, AS-LV-NC group, AS-LV-miR-155 group, and AS-LV-miR-155-RNAi group. All the mice were raised under standard conditions of room temperature, dark-light cycles in the Animal Center of the Affiliated Hospital of Qingdao University and fed with a high-fat diet (15\% fat and $0.25 \%$ cholesterol; KEAOXIELI, Beijing, China). Mice were anesthetized by intraperitoneal injection of pentobarbital sodium $(4 \mathrm{mg} / 100 \mathrm{~g}$ ) before the surgery at 8 -week-old. The right common carotid artery was inserted with a silicone ring to induce the formation of carotid atherosclerotic plaques in all $\mathrm{ApoE}^{-/-}$mice. The lentivirus was injected into the tail vein $48 \mathrm{~h}$ after the surgery to set up overexpression (LV-miR-155) and low expression (LV-miR-155-RNAi) models. After 8 weeks, all the mice were euthanized using pentobarbital sodium $(15 \mathrm{mg} / 100 \mathrm{~g})$ to obtain carotid artery samples. All animal experiments were approved by the Medical Ethics Committee of The Affiliated Hospital of Qingdao University and performed in compliance with legal and institutional guidelines. Efforts were performed to lessen animals' suffering.

\section{Histological observation and immunobistochemistry (IHC)}

After animals were sacrificed, the carotid arteries 
were at once extracted and respectively fixed with 4\% paraformaldehyde for $24 \mathrm{~h}$ and embedded in paraffin. Carotid artery samples were cut into 5 -um sections for hematoxylin and eosin (HE) staining and IHC analysis. For IHC, the antigen was repaired after the paraffin section was dewaxed. The slices were blocked $5 \%$ goat serum and then cultured with primary antibodies of caspase-1 (1:200, BOSTER Biological Technology, Wuhan, China) and IL-1 $\beta$ (1:200, Abcam, USA) at $37^{\circ} \mathrm{C}$ for $1 \mathrm{~h}$, after washing three times with $\mathrm{PBS}$ the sections were incubated with secondary antibody at $37{ }^{\circ} \mathrm{C}$ for $1 \mathrm{~h}$. DAB Horseradish Peroxidase Color Development Kit (Solarbio, China) was used to staining the sections. Cell nucleus was dyed with Mayer's hematoxylin solution. The staining slides were assessed with a light microscope at 200× magnification.

\section{Cell culture and preparation}

THP-1 monocytes were obtained from the American Type Culture Collection (ATCC, Manassas, USA). The cells were cultured in RPMI-1640 containing $10 \%$ fetal bovine serum (FBS; Thermo Scientific HyClone, Logan, USA), $100 \mu \mathrm{g} / \mathrm{mL}$ streptomycin and $100 \mathrm{U} / \mathrm{mL}$ penicillin (Invitrogen, Carlsbad, USA) and were incubated at $37{ }^{\circ} \mathrm{C}$ under $5 \% \mathrm{CO}_{2}$. Phorbol 12 -myristate 13 -acetate (PMA; Sigma-Aldrich, St. Louis, USA) was applied to induce the differentiation of THP-1 monocytes into macrophages. THP -1 cells $\left(1 \times 10^{6}\right.$ cells $)$ were differentiated into macrophages in 6-well plates with $2 \mathrm{~mL}$ complete medium containing $100 \mathrm{ng} / \mathrm{mL}$ PMA for $48 \mathrm{~h}$. The differentiated macrophages were then treated with $50 \mu \mathrm{g} / \mathrm{mL}$ ox-LDL for $24 \mathrm{~h}$ (18). The supernatants were collected in $1.5 \mathrm{~mL}$ tubes for ELISA, and the plates were washed 3 times with PBS and were prepared for total RNA and protein extraction.

\section{Oil Red O staining}

The macrophages in the plates were rinsed with PBS and were fixed with $4 \%$ paraformaldehyde solution for $30 \mathrm{~min}$. The cells were washed with PBS again and were rinsed with isopropanol for $5 \mathrm{~s}$. Then, the cells were stained in Oil Red $\mathrm{O}$ solution for $30 \mathrm{~min}$, eluted into isopropanol for $5 \mathrm{~s}$ and rinsed with PBS.

\section{Intervention of miR-155 expression and the transfection of cells}

Twenty-four hours before transfection, the differentiated macrophages were incubated in RPMI-1640 containing $10 \%$ FBS without antibiotics in 24-well plates. Shortly before transfection, the culture medium was changed to Opti-MEM I Reduced Serum Medium from GIBCO BRL (Grand Island, NY, USA). The macrophages were transfected with the complex of Lipofectamine 2000 (Invitrogen, Carlsbad, USA) and miR-155 mimics, inhibitors, or the $\mathrm{NC}$ at $50 \mathrm{nM}$ (the mixture ratio was 1:1). Six hours later, the fluorescence expression conditions of cell transfection were observed with a fluorescent microscope. Then, the cells were cultured in fresh, normal complete medium containing $50 \mu \mathrm{g} / \mathrm{mL}$ ox-LDL with or without the ERK pathway inhibitor U0126 (Sigma-Aldrich, St. Louis, MO, USA).

\section{Reverse transcription-quantitative PCR (qRT-PCR)}

The total RNA was extracted from arteries and macrophages using the TRIzol Reagent (Invitrogen, Carlsbad, USA) according to the instructions provided by the manufacturer and was stored at $-80{ }^{\circ} \mathrm{C}$. The RNA samples were converted into cDNA using the miRNA FirstStrand Synthesis Kit (Takara, Dalian, China) according to the manufacturer's protocol. The cDNAs samples were used for qRT-PCR with the Mir-X ${ }^{\mathrm{TM}}$ miRNA qRT-PCR SYBR ${ }^{\circledR}$ Kit (Takara, Dalian, China). The miRNA-155 primer sequence for RT-PCR was TGC GGT TAA TGC TAA TCG TGA TAG G (Takara, Dalian, China). All reactions ran as follows: denaturation $\left(95^{\circ} \mathrm{C}\right.$ for $\left.10 \mathrm{~min}\right)$, denaturation $\left(95^{\circ} \mathrm{C}\right.$ for $\left.10 \mathrm{~s}\right)$, annealing and extension $\left(60^{\circ} \mathrm{C}\right.$ for $\left.1 \mathrm{~min}\right)$ for 40 cycles. The relative miRNA expression levels were quantified by the comparative $\mathrm{Ct}$ method $\left(2^{-\Delta \Delta \mathrm{Ct}}\right)$ with $\mathrm{U} 6$ as an internal control.

\section{Western blot (WB) analysis}

The arterial tissue after grinding and cells were suspended in RIPA lysis buffer for $30 \mathrm{~min}$. After centrifugation $(10,000 \mathrm{rpm}$ for $10 \mathrm{~min})$, the protein concentration of the supernatants was quantified using the BCA Protein Assay Kit (Solarbio, Beijing, China). The protein samples were then incubated at $95^{\circ} \mathrm{C}$ for $10 \mathrm{~min}$. The denatured proteins (20 $\mu \mathrm{g} / \mathrm{group}$ ) were segregated by sodium dodecyl sulfatepolyacrylamide gel electrophoresis (SDS-PAGE) and were electrophoretically transferred onto polyvinylidene difluoride (PVDF) membranes (Millipore, Billerica, USA). Subsequently, the membranes were shaken and blocked with $5 \%$ bovine serum albumin (BSA) dissolved in Tris-buffered 


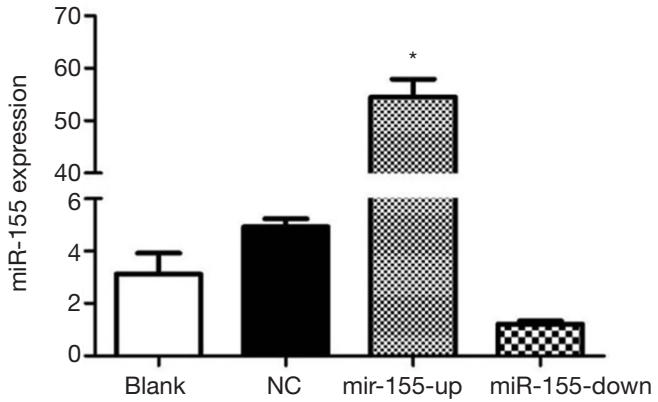

Figure 1 The expression of miR-155 on carotid AS of ApoE ${ }^{-/-}$ mice. The miR-155 expression in four groups of $\mathrm{ApoE}^{-/}$mice. *, $\mathrm{P}<0.05$, compared with the NC group. NC, negative control; AS, atherosclerosis.

saline-Tween (TBS-T) for $2 \mathrm{~h}$ at room temperature, followed by incubation in the primary antibody at $4{ }^{\circ} \mathrm{C}$ overnight. After washing the membranes with $0.1 \%$ TBS-T, the membranes were treated with the horseradish peroxidase (HRP)-labeled secondary polyclonal antibodies (Elabscience Biotechnology, Wuhan, China) for $2 \mathrm{~h}$ at room temperature. The bands were detected with an enhanced chemiluminescence kit (EMD Millipore, Billerica, MA, USA). The relative band intensity was analyzed by ImageJ software (National Institutes of Health, Bethesda, MD, USA).

Enzyme-linked immunosorbent assay (ELISA) for determining the levels of $I L-1 \beta$

The IL-1 $\beta$ and IL-18 levels in the cell supernatants were measured using ELISA kits (R\&D Systems, Minneapolis, USA).

\section{Statistical analysis}

All results are presented as the means \pm standard error of the mean (SEM). Unpaired, two-tailed Student's $t$-tests were used for comparing the 2 groups. Differences between multiple groups were determined by one-way ANOVA, followed by Bonferroni's multiple comparison test to determine the differences between the groups. The values of $\mathrm{P}<0.05$ were considered to have a significant difference. The statistical analysis was performed using IBM SPSS software (version 21.0).

\section{Results}

\section{The effect of miR-155 on carotid $A S$ of $A p o E^{-/-}$mice}

$\mathrm{ApoE}^{-/-}$mice were fed with a high-fat diet, and the right common carotid arteries were inserted with silicone cannula at 8-week-old to induce the formation of carotid AS plaques. The lentiviral vector ( $\mathrm{LV})$ was transfected by intravenous tail injection to interfere the expression of miR155. We detected the expression of miR-155 in carotid tissues and found that the LV-miR-155 significantly upregulate the miR-155 expression, and the LV-miR-155RNAi down-regulate the miR-155 expression $(\mathrm{P}<0.05$, Figure 1).

The plaque areas of right common carotid arteries were examined by HE stains. The results showed that there were varying degrees of plaque formation in all groups. When compared with the blank group, there was no distinct difference in terms of intravascular plaques in the $\mathrm{NC}$ group, while the intravascular plaques in the miR-155up group were remarkably increased. The carotid artery wall was significantly thickened with a large amount of inflammatory cell infiltration and accumulation of foam cells. Plaques in the miR-155-down group showed the opposite trend (Figure 2A).

Furthermore, we used IHC to preliminarily detect the expression of caspase- 1 and IL- $1 \beta$ in carotid tissues of mice (Figure 2B,C). We found that the expression of caspase-1 and IL-1 $\beta$ in the miR-155-up group was the highest of the four groups. There was no distinct difference between the blank and NC group, and the expression of caspase- 1 and IL- $1 \beta$ was lower in the miR-155-down group compared with the blank group. What is more, Western blot (WB) results also showed that NLRP3, caspase- 1 , and IL- $1 \beta$ were much more highly expressed in the miR-155-up group, compared with the $\mathrm{NC}$ group $(\mathrm{P}<0.05$, Figure $2 D)$.

\section{The effect of ox-LDL on the activation of the NLRP3 inflammasome in THP-1 macrophages}

The visual observation of Oil Red $\mathrm{O}$ staining revealed that many lipid droplets had accumulated in the THP1 macrophages after a $24 \mathrm{~h}$ incubation with $50 \mu \mathrm{g} / \mathrm{mL}$ oxLDL (Figure 3A), which shows foam cell formation. The NLRP3-related proteins, including NLRP3, caspase-1, IL- $1 \beta$, and IL-18, in ox-LDL-induced macrophages, were 
A

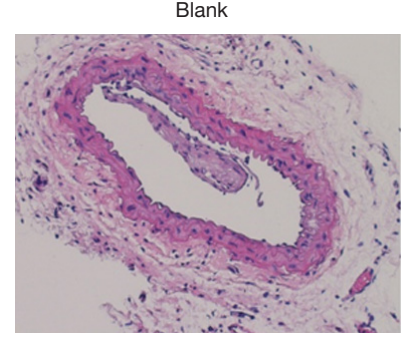

B

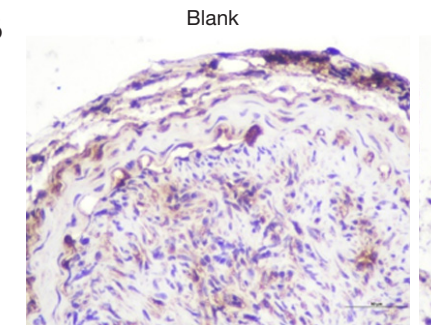

C

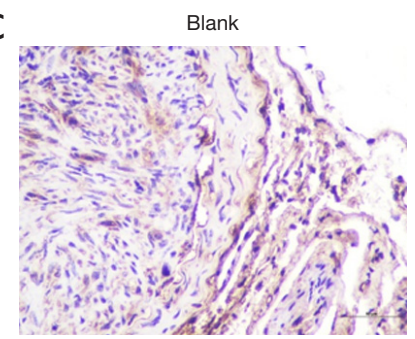

NC

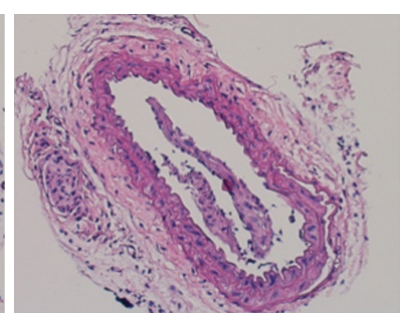

$\mathrm{NC}$

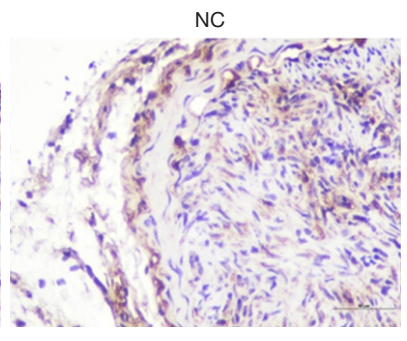

miR-155-up
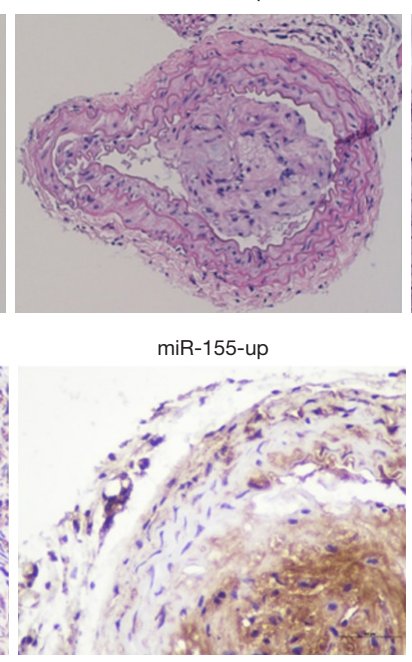

miR-155-down

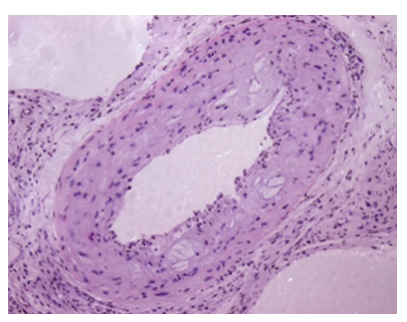

miR-155-down

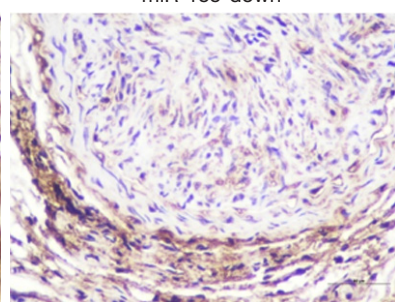

NC

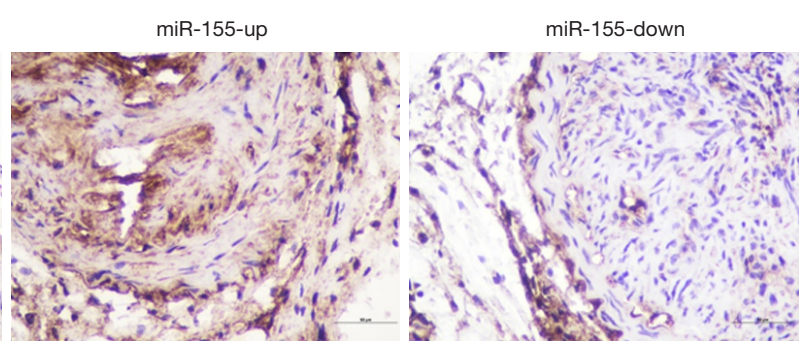

D
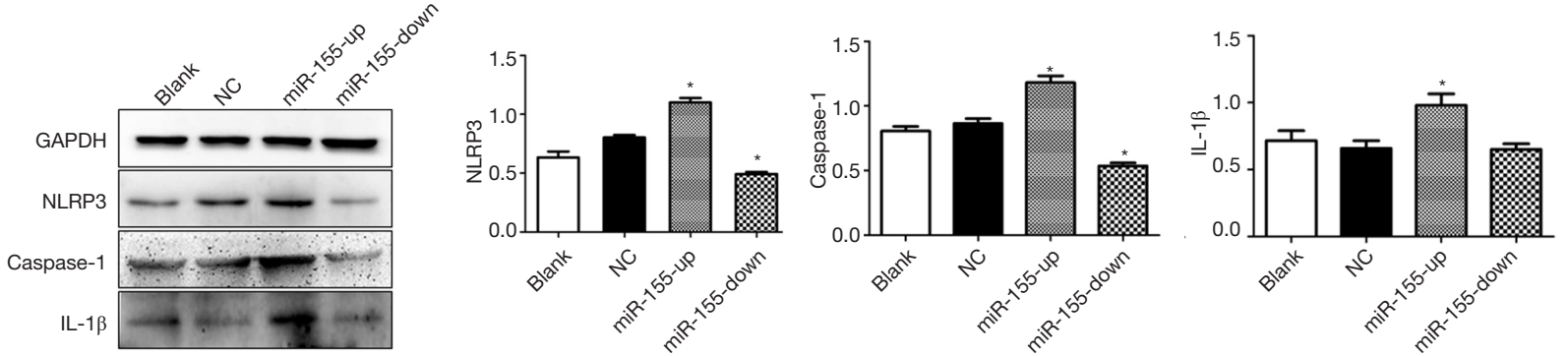

Figure 2 The effect of miR-155 on carotid AS of $\mathrm{ApoE}^{-/-}$mice. (A) Detection of AS lesion by HE stains revealed that plaque lesion is aggravated by miR-155 elevation ( $\times 200)$; (B,C) the results from the IHC suggested that expression of caspase- 1 (B) and IL- $1 \beta$ (C) in the carotid arteries is prominently increased by miR-155 elevation ( $\times 400$ ); (D) the expression of NLRP3, caspase-1, and IL-1 $\beta$ in the carotid arteries was detected by Western blot and the gray value ratio of WB image was calculated for statistical analyses using ImageJ. The data are representative images and are presented as the means $\pm \mathrm{SD},{ }^{*}, \mathrm{P}<0.05$, compared with the NC group. NC, negative control; AS, atherosclerosis; IHC, immunohistochemistry; HE, hematoxylin and eosin.

overexpressed compared with those in the control group. We also detected the increased phosphorylation of ERK1/2 and NF- $\kappa \mathrm{B}$ in the ox-LDL-induced macrophages $(\mathrm{P}<0.05$, Figure $3 B$ ). In addition, the IL- $1 \beta$ and IL-18 concentrations in supernatants were also significantly increased in the oxLDL-treated groups, which was consistent with the $\mathrm{WB}$ analysis $(\mathrm{P}<0.05$, Figure $3 C)$.

\section{The ERK1/2 pathway is involved in the activation of the $N L R P 3$ inflammasome in response to ox-LDL stimulation}

To evaluate whether the ERK1/2 pathway is involved in the NLRP3 inflammasome activation response to ox-LDL, THP-1 macrophages were incubated in $50 \mu \mathrm{g} / \mathrm{mL}$ oxLDL with or without $10 \mu \mathrm{M}$ of the ERK inhibitor U0126 for $24 \mathrm{~h}$. The phosphorylation of ERK1/2 was inhibited 
A

B
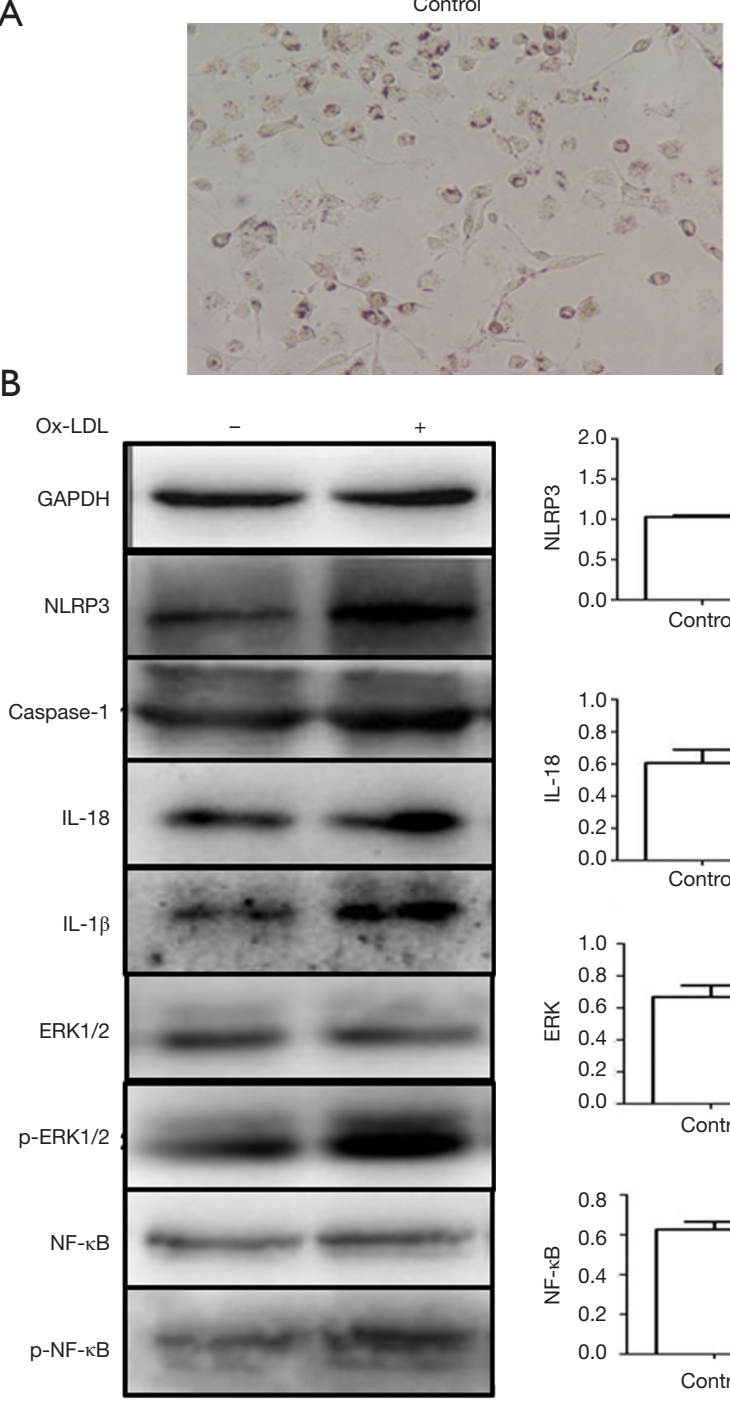

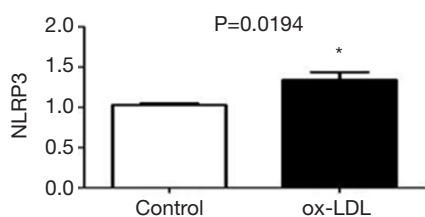

ox-LDL
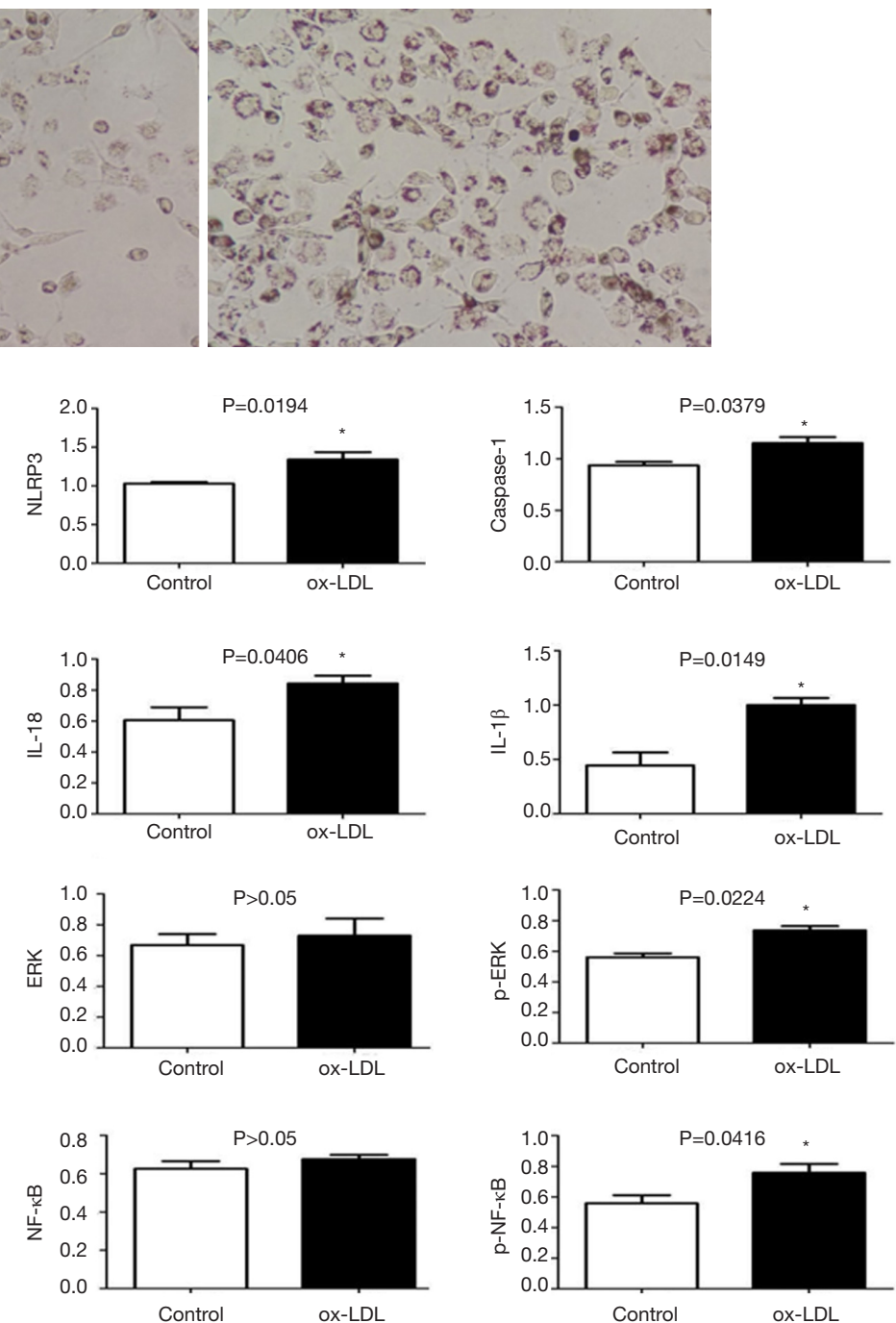

C
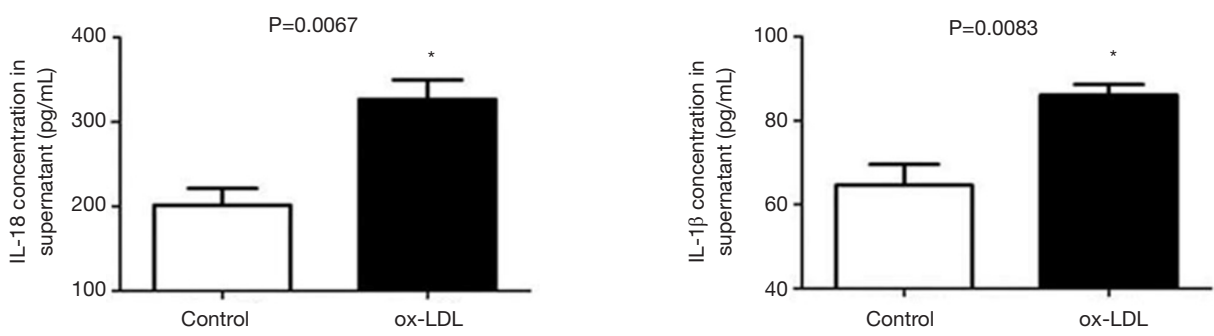

Figure 3 The effects of ox-LDL on THP-1 macrophages. (A) The ox-LDL group had higher lipid-loaded macrophage and foam cell formation levels; (B) the expression of NLRP3-related proteins, such as ERK1/2 and NF- $\mathrm{BB}$ in ox-LDL-induced THP-1 macrophages, was detected by Western blot and the gray value ratio was calculated for statistical analyses using ImageJ; (C) the IL-1 $\beta$ and IL-18 concentrations in the cell supernatant are shown. The data are representative images and are presented as the means $\pm \mathrm{SD}$. ${ }^{*}, \mathrm{P}<0.05$, compared with the blank control group. ox-LDL, oxidized low-density lipoprotein. 


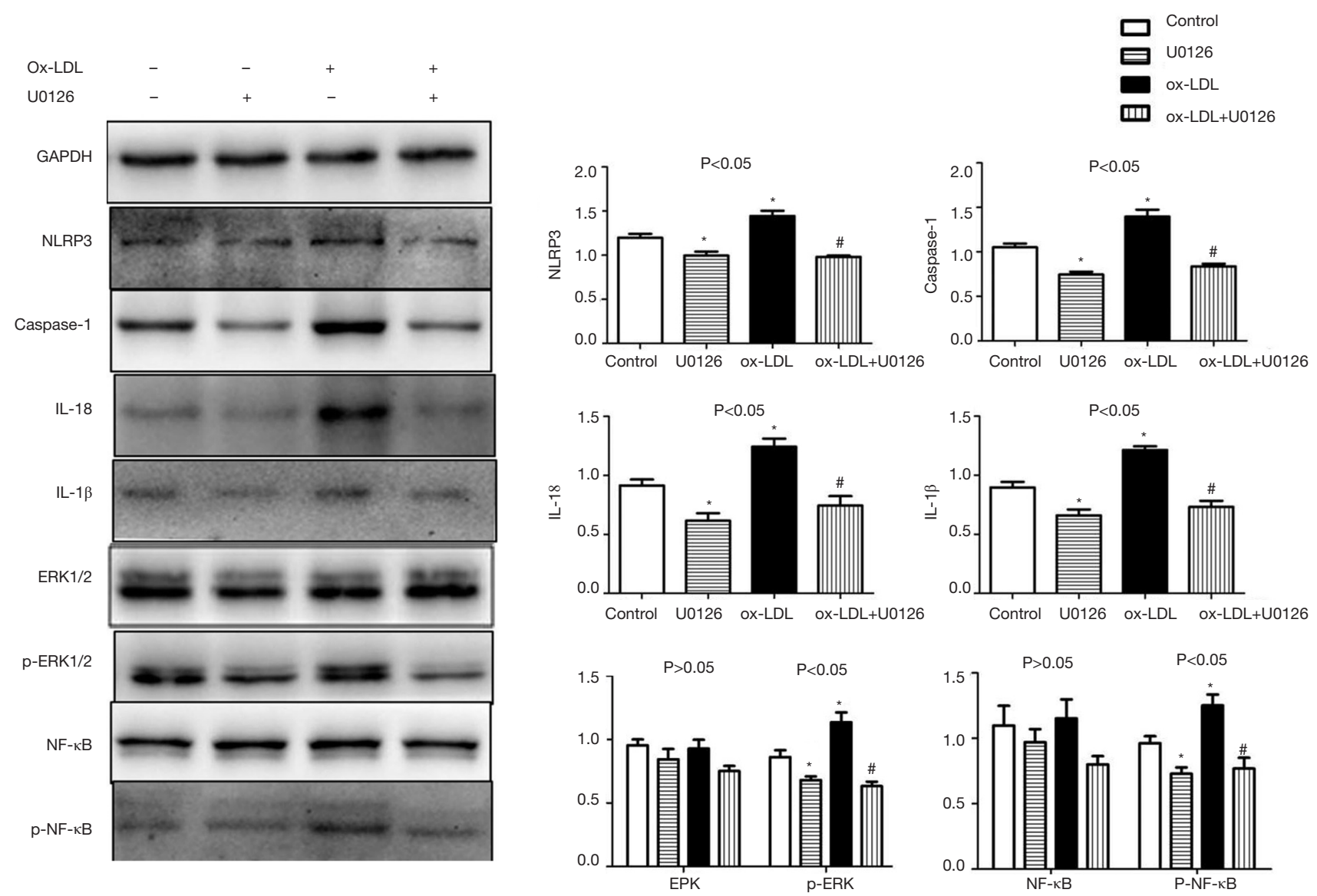

Figure 4 The ERK pathway inhibitor U0126 blocked the activation of the NLRP3 inflammasome, which had been induced by ox-LDL. U0126 effectively suppressed the expression of NLRP3 inflammasome-related proteins and the phosphorylation of ERK1/2 and NF-kB. The gray value ratio was calculated for statistical analyses using Image J. The data are representative images and are presented as the means \pm SD. *, $\mathrm{P}<0.05$, compared with the blank control group; ${ }^{*}, \mathrm{P}<0.05$, compared with the ox-LDL group. ox-LDL, oxidized low-density lipoprotein.

by U0126 and was accompanied by a later decrease in the phosphorylation of NF- $\mathrm{BB}(\mathrm{P}<0.05$, Figure 4). Meanwhile, the expression was drastically down-regulated for all examined NLRP3-related proteins (NLRP3, caspase-1, IL-1 $\beta$, and IL-18) under the influence of U0126 $(\mathrm{P}<0.05$, Figure 4).

\section{Effects of miR-155 on the ox-LDL-induced activation of the NLRP 3 inflammasome and the ERK1/2 pathway}

We saw the increased miRNA-155 expression in macrophages incubated with ox-LDL $(\mathrm{P}<0.05$, Figure $5 A)$. However, it is not known whether this up-regulated expression of miRNA-155 is associated with NLRP3 inflammasome activation induced by ox-LDL. To answer this question, we used miRNA mimics and inhibitors to interfere with miRNA-155 expression. The transfection of miR-155 mimics significantly up-regulated the expression of miR-155 by 40 -fold, while the miR-155 inhibitor group showed approximately a quarter of the expression compared with its NC group $(\mathrm{P}<0.0001$, Figure $5 B)$.

According to the WB analysis, the overexpression of miR-155 significantly increased the expression of NLRP3, caspase-1, IL-1 $\beta$, and IL-18 in macrophages, as well as the IL- $1 \beta$ and IL-18 concentrations in the medium. The downregulated expression of miR-155 reduced the expression of caspase- 1 and IL-18 in the macrophages and reduced the concentrations of IL- $1 \beta$ and IL-18 in the supernatant $(\mathrm{P}<0.05$, Figure 6). These results suggest that miR-155 promoted the activation of the NLRP3 inflammasome and that the down-regulated miR-155 restrained the release of IL-1 $\beta$ and IL-18. We also observed that the expression 
A

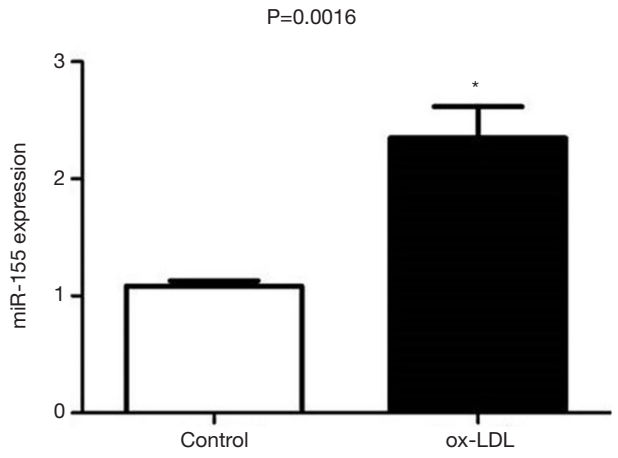

B

B $\quad P<0.0001$

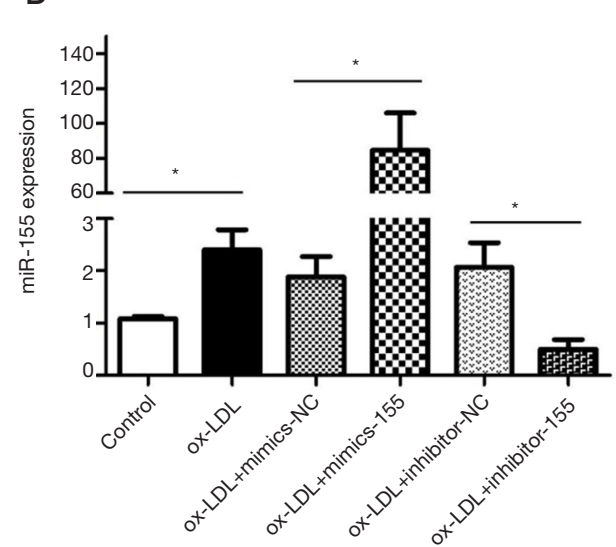

Figure 5 The expression level of miR-155 and the fluorescent observation of transfection. (A) The expression level of miR-155 was detected by qRT-PCR. All the values of RT-PCR were calculated by the comparative Ct method $\left(2^{-\Delta \Delta C t}\right)$; (B) the expression of miR-155 was detected after the transfection of mimics or inhibitors and the incubation of ox-LDL for $24 \mathrm{~h}$. The data are presented as the means $\pm \mathrm{SD}$. * $\mathrm{P}<0.05$, compared with the blank control group. qRT-PCR, quantitative reverse transcription polymerase chain reaction.

of phosphor-ERK1/2 and phosphor-NF- $\mathrm{KB}$ was higher in the miR-155 mimic group and was lower in the miR-155 inhibitor group than the expression levels in their respective control groups $(\mathrm{P}<0.05$, Figure 6), which indicated that miR-155 also activated the ERK1/2 pathway.

The overstimulation effect of miR-155 on the activation of the NLRP3 inflammasome is through the ERK/NF-кB pathway

We studied whether, during ox-LDL treatment, the ERK1/2 pathway mediates miR-155-induced NLRP3 inflammasome activation. The macrophages were treated with ox-LDL and were incubated with or without U0126 based on the up-regulation of miR-155. The WB analysis revealed that U0126 blocked the miR-155-induced excessive activation of the NLRP3 inflammasome: the expression levels of NLRP3, caspase-1, IL-1 $\beta$ and IL-18 in macrophages were all reduced in the miR-155 mimic + U0126 group $(\mathrm{P}<0.05$, Figure $7 A, B)$, accompanied by a decrease in the phosphorylation of ERK1/2 and NF- $\kappa \mathrm{B}(\mathrm{P}<0.05$, Figure $7 C)$. And the IL-1 $\beta$ and IL-18 concentrations in the supernatant showed the same trend $(\mathrm{P}<0.05$, Figure $7 D)$.

\section{Discussion}

In the present study, we found that in carotid AS mouse model, up-regulation of miR-155 can aggravate AS lesions and promote the activation of NLRP 3 inflammasome and the release of inflammatory factor IL-1 $\beta$. According to the results of in vitro experiment, ox-LDL induces NLRP3 inflammasome-associated protein expression via the ERK1/2 pathway and promotes miR-155 expression in macrophages. Further intervention studies suggest that miR-155 overexpression promotes the ox-LDL-induced activation of the NLRP3 inflammasome and that the use of the ERK pathway inhibitor U0126 blocks the overactivation of the NLRP3 inflammasome caused by miR155. These findings suggest that miR-155 may regulate oxLDL-mediated NLRP3 inflammasome activation via the ERK1/2 pathway and affect the formation of AS plaques in carotid arteries.

MiR-155 is an ancient regulator of the immune system and is induced by activation of $\mathrm{B}$ and $\mathrm{T}$ lymphocytes, as well as in cells of the innate immune system such as monocytes, macrophages (19), and DC (20). MiR-155 is able to shape the transcriptome of activated myeloid and lymphoid cells, consequently controlling diverse biological functions such as in hematopoietic lineage differentiation, immunity, inflammation, and autophagy $(21,22)$. Existing research shows that miR-155 can play both positive and negative roles in the control of inflammation (23) and plays a crucial role in cardiovascular diseases such as coronary (24) and cerebral artery disease (25), heart failure (26) and diabetic heart disease (27). Previous in vivo studies have found that high-fat diet-fed miR-155-deficient $\mathrm{ApoE}^{-/-}$ 
A

$\begin{array}{rcccccc}\text { Ox-LDL } & - & + & + & + & + & + \\ \text { miR-155 mimics } & - & - & - & + & - & - \\ \text { miR-155 inhibitor } & - & - & - & - & - & + \\ \text { Mimics NC } & - & - & + & - & - & - \\ \text { Inhibitor NC } & - & - & - & - & + & -\end{array}$

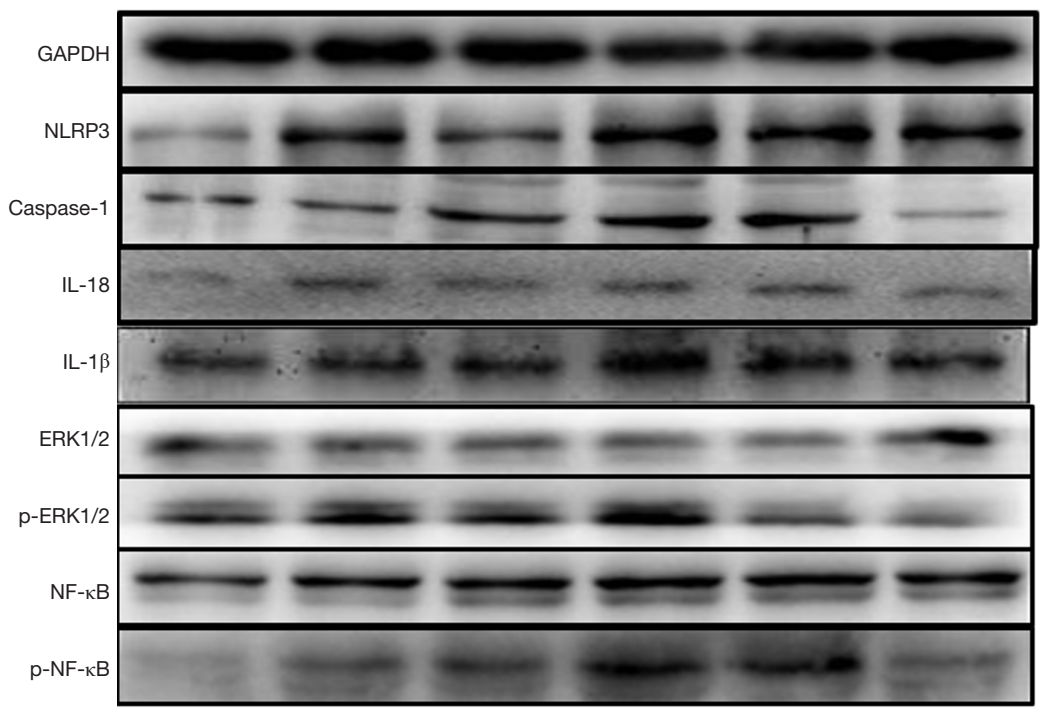

B
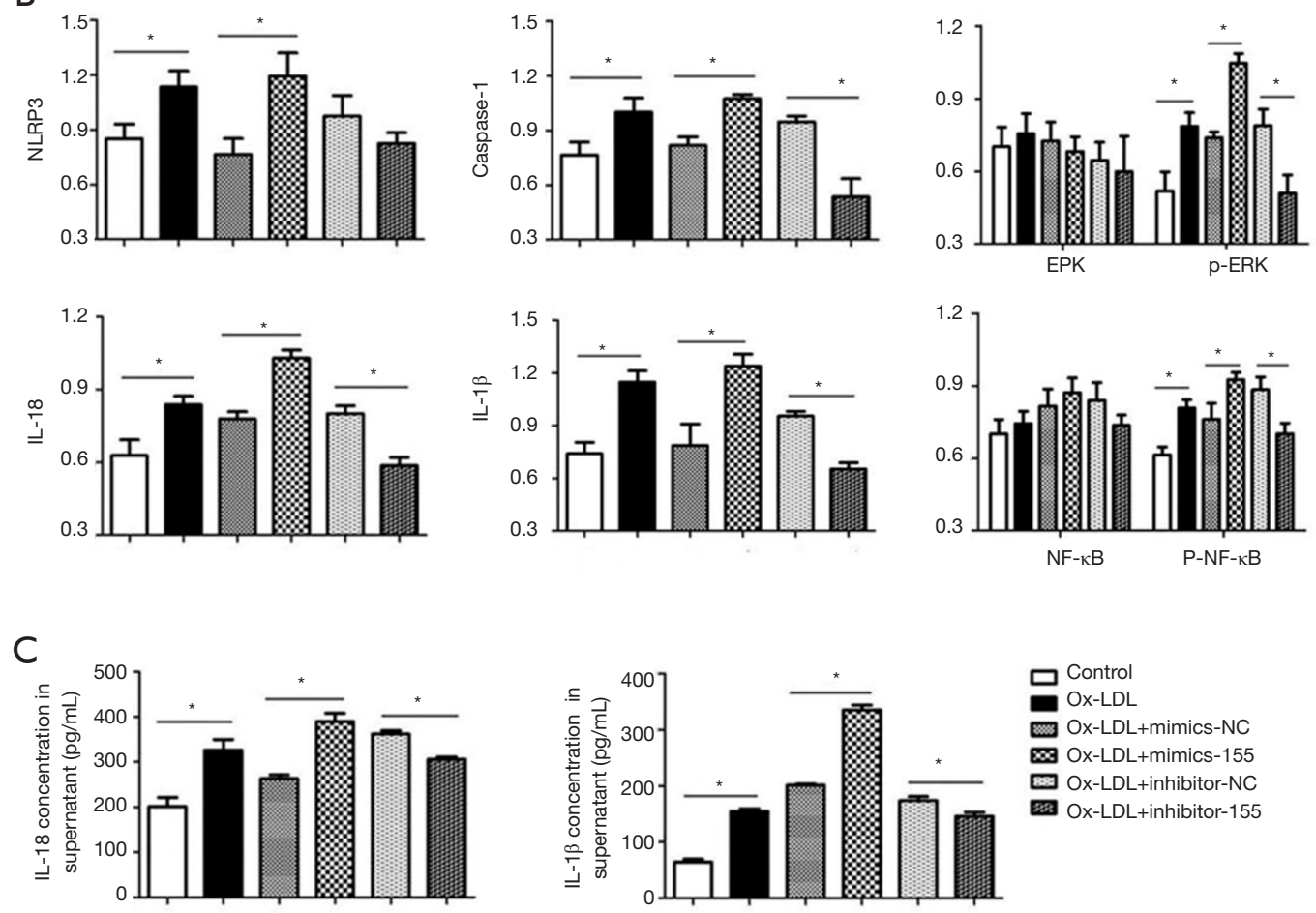

Figure 6 MiR-155 regulates the activation of the NLRP3 inflammasome in THP-1 macrophages and is associated with the ERK1/2 pathway. $(\mathrm{A}, \mathrm{B})$ The up-regulation of miR-155 significantly promotes the expression of NLRP3-related proteins and ERK/NF- $\mathrm{kB}$ phosphorylation; (C) the IL-1 $\beta$ and IL-18 concentrations in the cell supernatant are shown. The data are representative images and are presented as the means $\pm \mathrm{SD}$. * $\mathrm{P}<0.05$, compared between groups. 
A

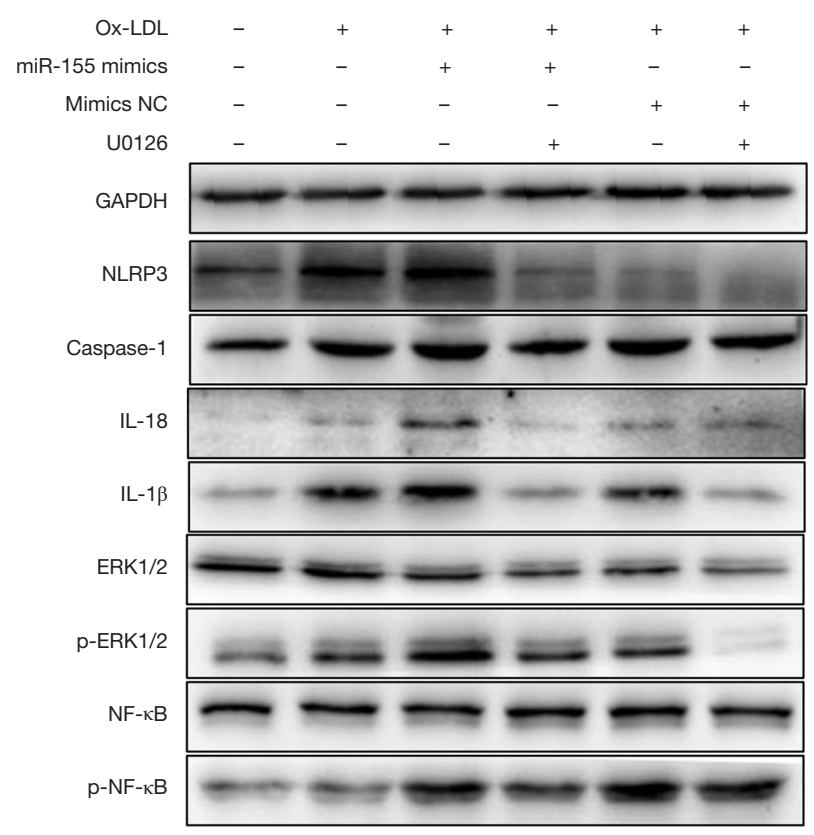

C
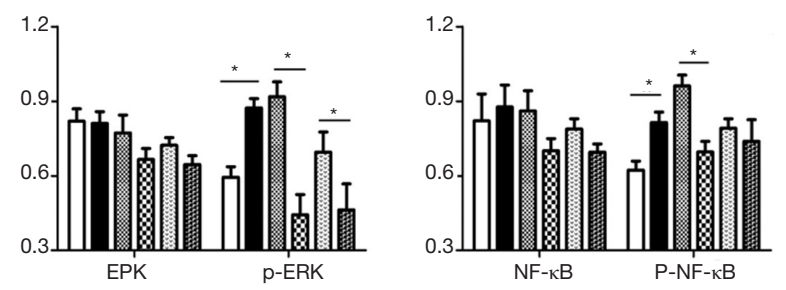

B
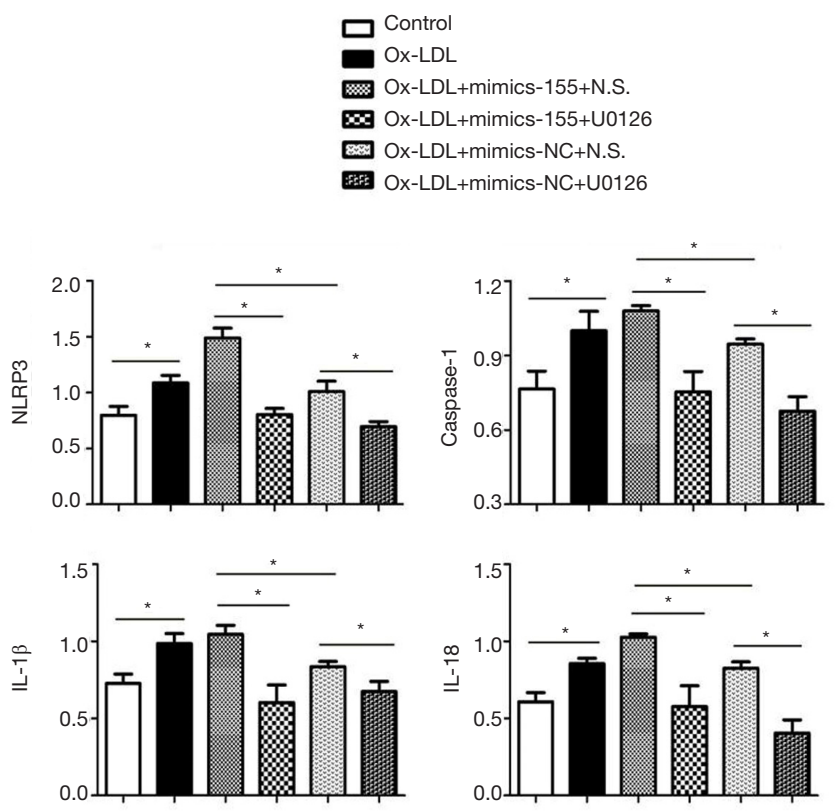

D
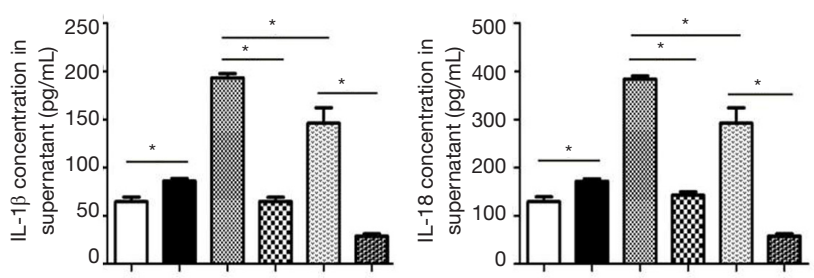

Figure 7 MiR-155 promotes the activation of the NLRP3 inflammasome via the ERK1/2 pathway in macrophages. U0126 can block the excessive activation of the NLRP3 inflammasome caused by the overexpression of miR-155. The data are representative images and are presented as the means $\pm \mathrm{SD} .{ }^{*}, \mathrm{P}<0.05$, compared between groups.

mice have a reduced plaque area and macrophage numbers, and the mechanism may be that miR-155 regulates the macrophage secretion of chemokine CCL2 through its target gene Bcl6 (28); the targeting of Bcl6 by miR-155 also inhibits pinocytosis and reduces the clearance of apoptotic macrophages, thereby further aggravating the inflammatory response (29). Our results from $\mathrm{ApoE}^{-/-}$mice are consistent with these findings (28-30) that elevated miR-155 could promote the AS formation and in the present study the underlying mechanism is probably associated with the NLRP3 inflammasome.

We choose ox-LDL-induced THP-1 macrophages as the cell model to further study these phenomena in vitro. OxLDL deposition and retention in plaques are the central events of AS. Ox-LDL can promote the expression of
NLRP3 and its downstream factor caspase- 1 and can induce the processing, maturation, and secretion of IL- $1 \beta$ and IL18 in the human monocyte cell line Thp-1 $(31,32)$. The present study found that after 24-h ox-LDL stimulation, the lipid droplets in THP-1 macrophages increased significantly and foam cells formed. Our results are consistent with those from earlier studies showing that the expression of NLRP3, caspase-1, IL-1 $\beta$ and IL-18 in model cells was up-regulated in response to ox-LDL stimulation.

According to existing research results, ox-LDL may activate the NLRP3 inflammasome in the following ways: (I) ox-LDL binds to the CD36 receptor on the surface of macrophages, promotes ROS production in cells, activates the downstream NLRP3 inflammasome and promotes IL- $1 \beta$ release (33); (II) ox-LDL up-regulates P2X7R- 
induced protein kinase $\mathrm{R}$ ( $\mathrm{PKR}$ ) phosphorylation in macrophages, promotes the formation of phosphorylated PKR and NLRP 3 complexes, and finally, causes NLRP3 inflammasome conformational changes and its activation (34); and (III) ox-LDL activates the NLRP3 inflammasome via TLR/NF- $\kappa \mathrm{B}$ and promotes the release of IL- $1 \beta$ by immune cells $(35,36)$. The use of the NF- $\kappa B-$ specific inhibitor Bay-11-7082 can significantly reduce IL$1 \beta$ protein expression levels. In the present study, we found that the ERK1/2 pathway phosphorylation was also elevated after ox-LDL stimulation in macrophages, in addition to the increase in NF- $\kappa \mathrm{B}$ phosphorylation, suggesting that the ERK1/2 pathway may also be an alternative upstream pathway of NF- $\kappa B$ activation induced by ox-LDL. The ERK1/2 pathway not only participates in the development of AS by regulating inflammatory molecules and oxidative stress but is also an important pathway for the activation of the NLRP3 inflammasome (37). The activation of the ERK1/2 pathway by LPS induces IL- $1 \beta$ mRNA expression and the protein expression of pro-IL-1 $\beta$ and NLRP3; this is the priming stage of NLRP3 inflammasome activation (38). Other studies further confirmed that LPS-induced NLRP3 inflammasome activation might be a posttranslational modification of the NLRP3 protein through the ERK signaling pathway, and deubiquitinases maybe its downstream targets (39). We used the ERK pathway inhibitor U0126 in model cells and found that not only the phosphorylation of the ERK pathway was significantly reduced, but also the phosphorylation level of the NF- $\mathrm{KB}$ pathway was decreased; in addition, the expression levels of NLRP3-related proteins were also significantly downregulated. These findings suggest that ox-LDL is likely to activate the NLRP3 inflammasome via the ERK1/2-NF- $\mathrm{KB}$ pathway.

In addition, we detected the expression level of miR155 in ox-LDL incubated macrophages and found that the expression of miR-155 was higher than that in the control cells. This result is in agreement with our previous results, where we found that miR-155 expression increased in $\mathrm{APOE}^{-/-}$mouse carotid AS plaques (15). In the early stages of AS, primary LDL and mildly oxidized LDL (mox-LDLs) promote endogenous miR-155 expression through LDL receptors, and this leads to macrophage activation and the secretion of chemokine. Subsequently, macrophages are promoted, as is neutrophil aggregation and infiltration, and this further promotes LDL oxidation through the positive feedback mechanism. At the same time, moxLDLs can also increase lipid uptake by LDL receptor- mediated macrophagia, which leads to the entry of LDLbound exogenous miR-155 into macrophages; this leads to a further increase in intracellular miR-155 levels (40-44) and forms a cascade reaction that continuously advances the inflammatory response. In addition, previous research also found that ox-LDL might up-regulate the miR-155 expression in DCs through scavenger receptor A (SRA) (45) and identified a negative feedback loop, miR-155-JNKSRA-miR-155, which could regulate the balance of miR155 expression.

The down-regulation of NLRP3 inflammasome-related proteins in $\mathrm{miR}-155^{-/-} \mathrm{DC}$ suggests that miR-155 may induce NLRP3 inflammasome activation (16). To investigate the effects of miR-155 on NLRP3 inflammasome activation in ox-LDL-induced THP-1 macrophages, we used miRNA mimics and inhibitors to up-regulate and down-regulate miR-155 expression and found that the overexpression of miR-155 can significantly promote the activation of the NLRP3 inflammasome response to oxLDL. The expression of NLRP3, caspase-1, IL-1 $\beta$, and IL18 is increased, and the concentrations of IL- $1 \beta$ and IL18 in the cell supernatant are also significantly increased. Compared with the expression levels in the NC group, the expression levels of the target proteins in the inhibitor group decreased, but the differences were not significant. The main reason may be that the miRNA inhibitor did not significantly reduce the expression level of intracellular miR-155 but only suppressed the function of miR-155 or increased its degradation. The above results suggest that overexpressed miR-155 can promote ox-LDL-induced NFRP3 inflammasome activation in macrophages. In

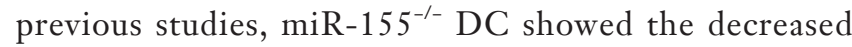
expression of MAPK pathway-related genes and decreased levels of ERK phosphorylation in addition to the expression of NLRP3-related molecules (16); this suggests that the miR-155 regulation of the NLRP3 inflammasome may be associated with the ERK/MAPK pathway. Therefore, to verify whether miR-155 in macrophages regulates the ox-LDL-induced inflammatory activation of NLRP3 through the ERK1/2 pathway, we applied the ERK pathway inhibitor U0126 and up-regulated the expression of miR155. It was found that U0126 can significantly block the ox-LDL-mediated activation of NLRP3 caused by the overexpression of miR-155, while in the normal expression group of miR-155, the blocking effect of U0126 on NLRP3 inflammasome activation was significantly attenuated. These results show that miR-155 exerts a regulatory effect on NLRP3 inflammasome activation in ox-LDL-induced 
macrophages via the ERK/NF-кB pathway.

\section{Conclusions}

In this study, we verified that elevated miR-155 activates the NLRP3 inflammasome and aggravate the carotid AS lesion in $\mathrm{ApoE}^{-/-}$mice, determined the regulatory effects of miR-155 on the NLRP3 inflammasome in macrophages for the first time, and further confirmed its pathway, ERK1/2. The results of this study will help to reveal the pathological mechanism of AS plaques and will have important significance in the analysis of the regulatory mechanism of miRNAs on the inflammatory response in AS plaques; this will supply a potential therapeutic target for the prevention and treatment of AS.

\section{Acknowledgments}

Funding: This study was supported by the National Natural Science Foundation of China (No. 81771259 and No. 81571112 ) and the Shandong Province Natural Science Foundation (ZR2017BH012).

\section{Footnote}

Conflicts of Interest: The authors have no conflicts of interest to declare.

Ethical Statement: The authors are accountable for all aspects of the work in ensuring that questions related to the accuracy or integrity of any part of the work are appropriately investigated and resolved. The study was approved by the Medical Ethics Committee of the Affiliated Hospital of the Qingdao University (No. 2017-81771259).

\section{References}

1. Ross R. Atherosclerosis--an inflammatory disease. N Engl J Med 1999;340:115-26.

2. Ma A, Pan X, Xing Y, et al. Elevation of serum CXCL16 level correlates well with atherosclerotic ischemic stroke. Arch Med Sci 2014;10:47-52.

3. Libby P. Inflammation in atherosclerosis. Nature 2002;420:868-74.

4. Pan X, Pang M, Ma A, et al. Association of TRAIL and Its Receptors with Large-Artery Atherosclerotic Stroke. PloS One 2015;10:e136414.

5. Ait-Oufella H, Taleb S, Mallat Z, et al. Recent advances on the role of cytokines in atherosclerosis. Arterioscler Thromb Vasc Biol 2011;31:969-79.

6. Li X, Zhang Y, Xia M, et al. Activation of Nlrp3 inflammasomes enhances macrophage lipid-deposition and migration: implication of a novel role of inflammasome in atherogenesis. PloS One 2014;9:e87552.

7. Paramel Varghese G, Folkersen L, Strawbridge RJ, et al. NLRP3 Inflammasome Expression and Activation in Human Atherosclerosis. J Am Heart Assoc 2016. doi: 10.1161/JAHA.115.003031.

8. Shi X, Xie WL, Kong WW, et al. Expression of the NLRP3 Inflammasome in Carotid Atherosclerosis. J Stroke Cerebrovasc Dis 2015;24:2455-66.

9. Zheng F, Xing S, Gong Z, et al. NLRP3 inflammasomes show high expression in aorta of patients with atherosclerosis. Heart Lung Circ 2013;22:746-50.

10. Wei $Y$, Nazari-Jahantigh $M$, Chan L, et al. The microRNA-342-5p fosters inflammatory macrophage activation through an Akt1- and microRNA-155dependent pathway during atherosclerosis. Circulation 2013;127:1609-19.

11. Li X, Kong D, Chen H, et al. miR-155 acts as an antiinflammatory factor in atherosclerosis-associated foam cell formation by repressing calcium-regulated heat stable protein 1. Sci Rep 2016;6:21789.

12. Sun HX, Zeng DY, Li RT, et al. Essential role of microRNA-155 in regulating endothelium-dependent vasorelaxation by targeting endothelial nitric oxide synthase. Hypertension 2012;60:1407-14.

13. Zhang E, Wu Y. Dual effects of miR-155 on macrophages at different stages of atherosclerosis: LDL is the key? Med Hypotheses 2014;83:74-8.

14. Yang Z, Zheng B, Zhang $Y$, et al. miR-155-dependent regulation of mammalian sterile 20-like kinase 2 (MST2) coordinates inflammation, oxidative stress and proliferation in vascular smooth muscle cells. Biochim Biophys Acta 2015;1852:1477-89.

15. Ma J, Yang $\mathrm{S}, \mathrm{Ma} A$, et al. Expression of miRNA-155 in carotid atherosclerotic plaques of apolipoprotein $\mathrm{E}$ knockout (ApoE(-/-)) mice and the interventional effect of rapamycin. Int Immunopharmacol 2017;46:70-4.

16. Chen S, Smith BA, Iype J, et al. MicroRNA-155-deficient dendritic cells cause less severe GVHD through reduced migration and defective inflammasome activation. Blood 2015;126:103-12.

17. Boaru SG, Borkham-Kamphorst E, Van de Leur E, et al. NLRP3 inflammasome expression is driven by NF-kappaB in cultured hepatocytes. Biochem Biophys Res Commun 
2015;458:700-6.

18. Wang T, Zhang L, Hu J, et al. Mst1 participates in the atherosclerosis progression through macrophage autophagy inhibition and macrophage apoptosis enhancement. J Mol Cell Cardiol 2016;98:108-16.

19. O'Connell RM, Taganov KD, Boldin MP, et al. MicroRNA-155 is induced during the macrophage inflammatory response. Proc Natl Acad Sci USA 2007;104:1604-9.

20. Feldman, C. Severe community-acquired pneumonia. Curr Opin Pulm Med 1997;3:98-104.

21. Vigorito E, Kohlhaas S, Lu D, et al. miR-155: an ancient regulator of the immune system. Immunol Rev 2013;253:146-57.

22. Zhang Z, Pan X, Yang S, et al. miR-155 Promotes oxLDL-Induced Autophagy in Human Umbilical Vein Endothelial Cells. Mediators Inflamm 2017;2017:9174801.

23. Tili E, Michaille JJ, Cimino A, et al. Modulation of miR155 and miR-125b levels following lipopolysaccharide/ TNF-alpha stimulation and their possible roles in regulating the response to endotoxin shock. J Immunol 2007;179:5082-9.

24. Wang R, Dong LD, Meng XB, et al. Unique MicroRNA signatures associated with early coronary atherosclerotic plaques. Biochem Biophys Res Commun 2015;464:574-9.

25. Virtue A, Johnson C, Lopez-Pastrana J, et al. MicroRNA-155 Deficiency Leads to Decreased Atherosclerosis, Increased White Adipose Tissue Obesity, and Non-alcoholic Fatty Liver Disease: A NOVEL MOUSE MODEL OF OBESITY PARADOX. J Biol Chem 2017;292:1267-87.

26. Marques FZ, Vizi D, Khammy O, et al. The transcardiac gradient of cardio-microRNAs in the failing heart. Eur J Heart Fail 2016;18:1000-8.

27. Costantino S, Paneni F, Luscher TF, et al. MicroRNA profiling unveils hyperglycaemic memory in the diabetic heart. Eur Heart J 2016;37:572-6.

28. Nazari-Jahantigh M, Wei Y, Noels H, et al. MicroRNA-155 promotes atherosclerosis by repressing Bcl6 in macrophages. J Clin Invest 2012;122:4190-202.

29. Wei Y, Zhu M, Corbalan-Campos J, et al. Regulation of Csf1r and Bcl6 in macrophages mediates the stage-specific effects of microRNA-155 on atherosclerosis. Arterioscler Thromb Vasc Biol 2015;35:796-803.

30. Tian FJ, An LN, Wang GK, et al. Elevated microRNA-155 promotes foam cell formation by targeting HBP1 in atherogenesis. Cardiovasc Res 2014;103:100-10.

31. Li WL, Hua LG, Qu P, et al. NLRP3 inflammasome: a novel link between lipoproteins and atherosclerosis. Arch Med Sci 2016;12:950-8.

32. Latz E, Xiao TS, Stutz A. Activation and regulation of the inflammasomes. Nat Rev Immunol 2013;13:397-411.

33. Liu W, Yin Y, Zhou Z, et al. OxLDL-induced IL-1 beta secretion promoting foam cells formation was mainly via CD36 mediated ROS production leading to NLRP3 inflammasome activation. Inflamm Res 2014;63:33-43.

34. Peng $\mathrm{K}$, Liu L, Wei D, et al. P2X7R is involved in the progression of atherosclerosis by promoting NLRP3 inflammasome activation. Int J Mol Med 2015;35:1179-88.

35. Chávez-Sánchez L, Garza-Reyes MG, Espinosa-Luna JE, et al. The role of TLR2, TLR4 and CD36 in macrophage activation and foam cell formation in response to oxLDL in humans. Hum Immunol 2014;75:322-9.

36. Björkbacka H, Kunjathoor VV, Moore KJ, et al. Reduced atherosclerosis in MyD88-null mice links elevated serum cholesterol levels to activation of innate immunity signaling pathways. Nat Med 2004;10:416-21.

37. Wang K, Yao Y, Zhu X, et al. Amyloid beta induces NLRP3 inflammasome activation in retinal pigment epithelial cells via NADPH oxidase- and mitochondriadependent ROS production. J Biochem Mol Toxicol 2017. doi: 10.1002/jbt.21887.

38. Liao PC, Chao LK, Chou JC, et al. Lipopolysaccharide/ adenosine triphosphate-mediated signal transduction in the regulation of NLRP3 protein expression and caspase1-mediated interleukin-1beta secretion. Inflamm Res 2013;62:89-96.

39. Ghonime MG, Shamaa OR, Das S, et al. Inflammasome priming by lipopolysaccharide is dependent upon ERK signaling and proteasome function. J Immunol 2014;192:3881-8.

40. Moore KJ, Tabas I. Macrophages in the pathogenesis of atherosclerosis. Cell 2011;145:341-55.

41. Tili E, Croce CM, Michaille JJ. miR-155: on the crosstalk between inflammation and cancer. Int Rev Immunol 2009;28:264-84.

42. Dorsett Y, McBride KM, Jankovic M, et al. MicroRNA-155 suppresses activation-induced cytidine deaminase-mediated Myc-Igh translocation. Immunity 2008;28:630-8.

43. Ghorpade DS, Leyland R, Kurowska-Stolarska M, et al. MicroRNA-155 is required for Mycobacterium bovis BCG-mediated apoptosis of macrophages. Mol Cell Biol 2012;32:2239-53.

44. Koch M, Mollenkopf HJ, Klemm U, et al. Induction 
of microRNA-155 is TLR- and type IV secretion system-dependent in macrophages and inhibits DNAdamage induced apoptosis. Proc Natl Acad Sci USA 2012;109:E1153-62.

Cite this article as: Yin R, Zhu X, Wang J, Yang S, Ma A, Xiao Q, Song J, Pan X. MicroRNA-155 promotes the ox-LDLinduced activation of NLRP3 inflammasomes via the ERK1/2 pathway in THP-1 macrophages and aggravates atherosclerosis in $\mathrm{ApoE}^{-/-}$mice. Ann Palliat Med 2019;8(5):676-689. doi: 10.21037/apm.2019.10.11
45. Yan $\mathrm{H}$, Wang S, Li Z, et al. Upregulation of miRNA-155 expression by OxLDL in dendritic cells involves JAK1/2 kinase and transcription factors YY1 and MYB. Int J Mol Med 2016;37:1371-8. 